\title{
Aortic intramural haematoma: current therapeutic strategy
}

\author{
M D Dake
}

Heart 2004;90:375-378. doi: 10.1136/hrt.2003.027631

O ver the last 10 years, intramural haematoma (IMH) of the aorta has become increasingly recognised as a pathological entity distinct from aortic dissection. ${ }^{1}$ Despite this enhanced appreciation and the resultant increase in the frequency of its diagnostic identification, a consensus regarding optimal management strategies for this disease has not been established. ${ }^{2}$ This is due in part to our incomplete understanding of a condition only first clearly established in the mid 1980s. As opposed to aortic dissection where we have developed a relatively comprehensive knowledge of the more common constellations of anatomic involvement and their clinical outcomes, with IMH we are now only at a stage of correlating insights gleaned from clinical series contributed by investigators from around the world. ${ }^{1-14}$ Many of theses observations have been presented in the prior discussions.

\section{MANAGEMENT STRATEGIES}

As the profile of clinical factors, imaging findings, acute outcomes, and long term results of various management strategies comes into sharper focus, some patterns are emerging and serve as a basis for establishing the initial standard treatment algorithms for IMH. One of the aspects of IMH, however, that confounds attempts to set indications for intervention, much less precise management techniques, is the wide variety of morphologic appearances of aortic IMH observed with common diagnostic imaging modalities, including transoesophageal echo (TOE) or magnetic resonance imaging/computed tomographic (MRI/CT) scans. Traditionally, IMH refers to haemorrhage contained within the medial layer of the aortic wall, and is distinguished from typical aortic dissection and penetrating atherosclerotic aortic ulcer by the absence of an associated tear in the intima or direct communication between the media and the aortic lumen.

Unfortunately, this seemingly straightforward differentiation is not always possible because of the rapid tempo of morphologic evolution noted frequently on sequential imaging exams of patients with IMH. Thus, depending on the exact time after the onset of symptoms when an imaging "snapshot" is performed, the appearance may be interpreted differently than an impression made from images obtained only hours before or after. In practical terms, because there are limitations in our ability to repeat imaging at frequent intervals, assignment of precise diagnostic labels in a dogmatic manner is not always possible (fig 1). Indeed, in many patients with acute aortic symptoms, a healthy bit of confusion between the related diagnoses of aortic dissection without intimal rupture, IMH, and penetrating atherosclerotic aortic ulcer with associated IMH is expected, and reflects the natural history of IMH.

In addition to the potential for diagnostic confusion that exists when imaging "samples" one point in the rapid tempo of morphological evolution characteristic of some IMH patients, other factors limit the facile development of criteria that would permit the blanket application of a general therapeutic strategy to manage patients with IMH. Some of the critical features identified in the medical literature that appear to influence the outcome of IMH, in terms of whether
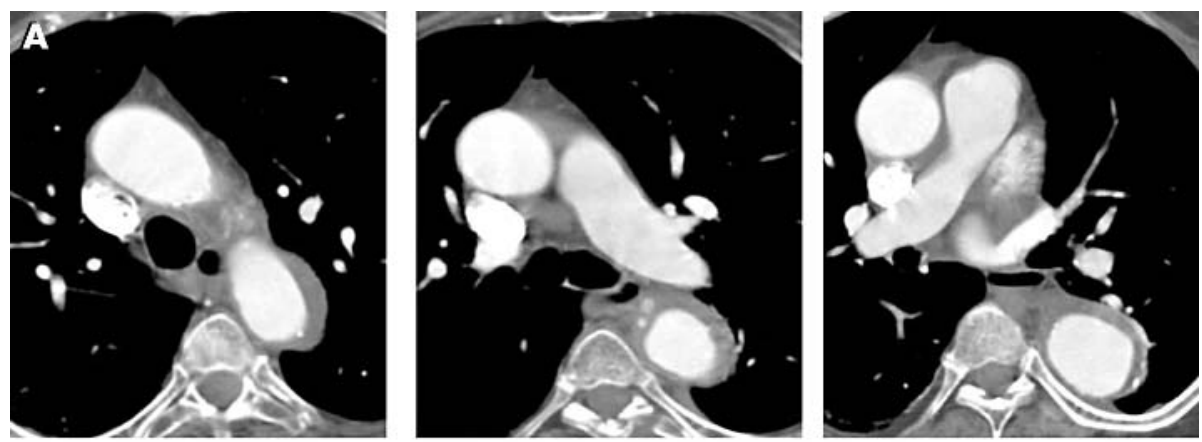

Figure 1 Evolution of type B intramural haematoma (IMH). Sequence of computed tomographic (CT) scans at the same axial levels performed initially (A) and three weeks (B) after the onset of symptoms. Note the intimal erosion evident distally on the most recent exam, the appearance of which could be misinterpreted as a penetrating aortic ulcer on an isolated scan without antecedent studies.
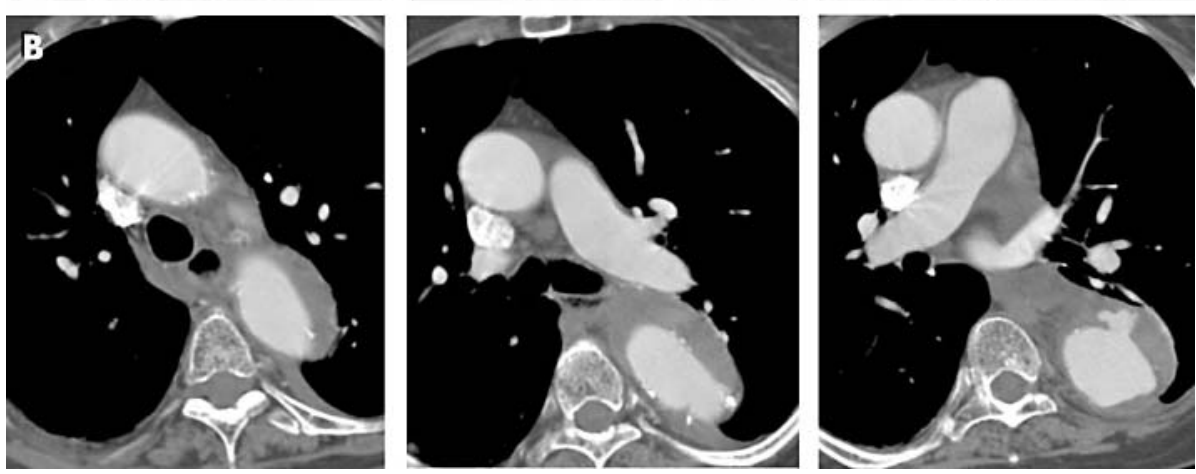

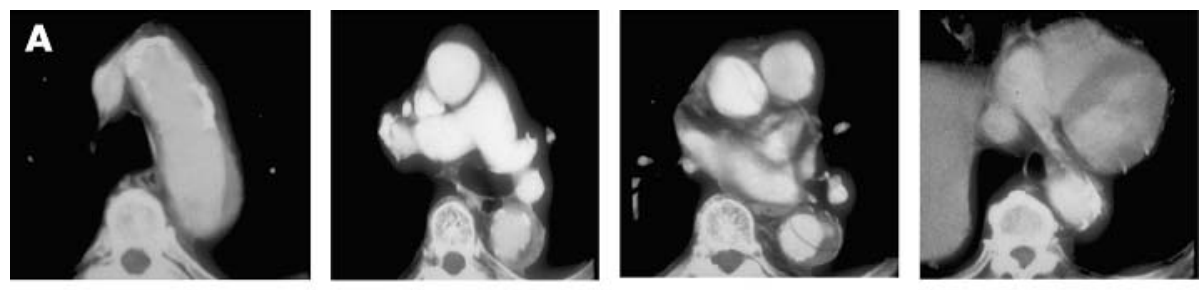

Figure 2 Evolution of type B IMH preand post-endovascular management of associated ulcer. (A) CT scan performed at the time of initial symptoms shows IMH and associated ulcer (third panel). (B) CT scan at the same level two weeks later when symptoms recurred. The ulcer is more prominent and a pleural effusion is evident.
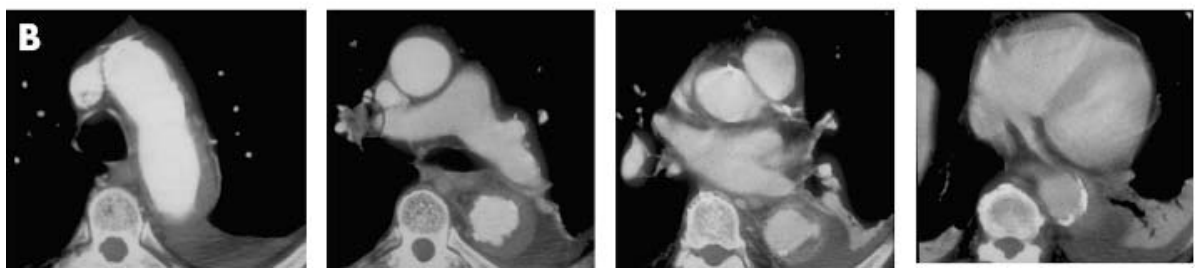

it has a complicated, progressive course or follows an uncomplicated, benign disease pattern include: the presence of acute symptoms at the time of imaging diagnosis; involvement of the ascending aorta, and presence of an associated penetrating ulcer or ulcer-like projection in the involved aortic segment. ${ }^{13-7}$

Born out of these initial clinical observations and despite the recognised limitations to formulating prognostic criteria for $\mathrm{IMH}$, recent attention focused on this entity over the last five years led to the identification of numerous new features of disease involvement that may allow a more reliable determination of the relative risk for an individual patient. It is hoped that these disease characteristics will be further refined in the future to better define those patients with an increased relative risk of progression to aortic dissection, rupture, aneurysm formation, IMH expansion, and those in which partial regression or complete resorption is likely.

\section{PREDICTORS OF DISEASE PROGRESSION}

Some of the current predictive factors of disease progression proposed for patients with IMH exclusively, without an associated ulcer or intimal erosion, include: involvement of the ascending aorta; maximum aortic diameter of $50 \mathrm{~mm}$ or greater on initial CT scan; persistent pain; progressive maximal aortic wall thickness; and enlarging aortic diameter. $^{36711}$ Other predictors of disease progression in patients with IMH and an associated aortic ulcer or intimal erosion include: interval increase of associated pleural effusion; recurrent pain; ulcer located in the ascending aorta or arch; initial maximum ulcer diameter of $20 \mathrm{~mm}$ or more; and initial maximum ulcer depth of $10 \mathrm{~mm}$ or greater. ${ }^{13}$
Given this background and with the recognition that roughly half the cases of IMH progress despite medical treatment while the others show spontaneous resolution of the process without clinical sequelae, a loosely held consensus regarding IMH management has coalesced around the traditional treatment algorithms applied to classic aortic dissection. ${ }^{23}$ Thus, one of the pivotal criteria used to direct treatment is based around the location of aortic involvement with the Stanford classification of aortic dissection commonly used to categorise IMH. In this regard, in many institutions, the standard treatment over the past decade for patients with type A IMH with or without an ulcer has been early surgical graft replacement.

Published 30 day mortality after surgery is reported to range from $10-50 \% .^{2{ }^{39-11}}$ Despite the uncertain risk of operative repair, it is a firmly held belief that for acute IMH presenting within a few hours of symptom onset and involving the ascending aorta, observation and medical treatment are far more dangerous than surgery. ${ }^{2}$ 9-11 Alternatively, other investigators have advocated conservative treatment for type A IMH and report favourable results without progression to classic dissection, rupture, tamponade, or compression of coronary ostia. ${ }^{42-14}$ Song and colleagues suggest that medical treatment initially with frequent imaging follow up and elective surgery in cases that develop complications is a prudent strategy. ${ }^{8}$ These conservative approaches currently, however, remain minority opinions for dealing with acutely symptomatic patients.

\section{ASYMPTOMATIC PATIENTS}

Conversely, in asymptomatic patients or those beyond 48 hours from the onset of symptoms with IMH located in the
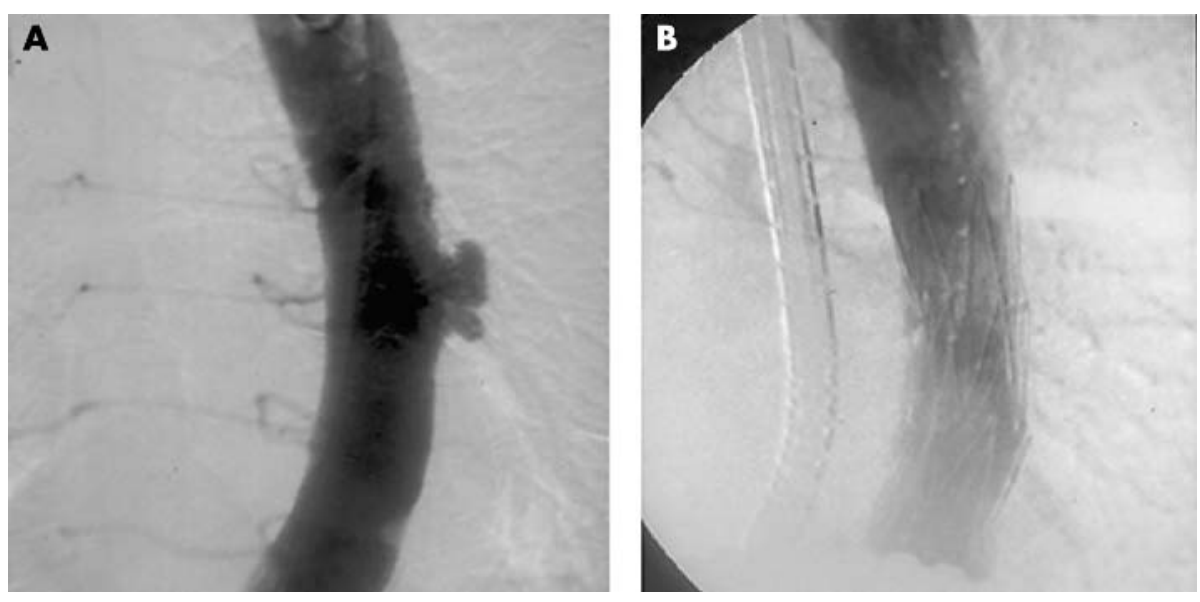

Figure 3 Evolution of type B IMH preand post-endovascular management of associated ulcer. (A) Aortogram shows aortic ulcer pre-intervention. (B) Aortogram after stent-graft deployment across ulcer and limited segment of $\mathrm{IMH}$. 

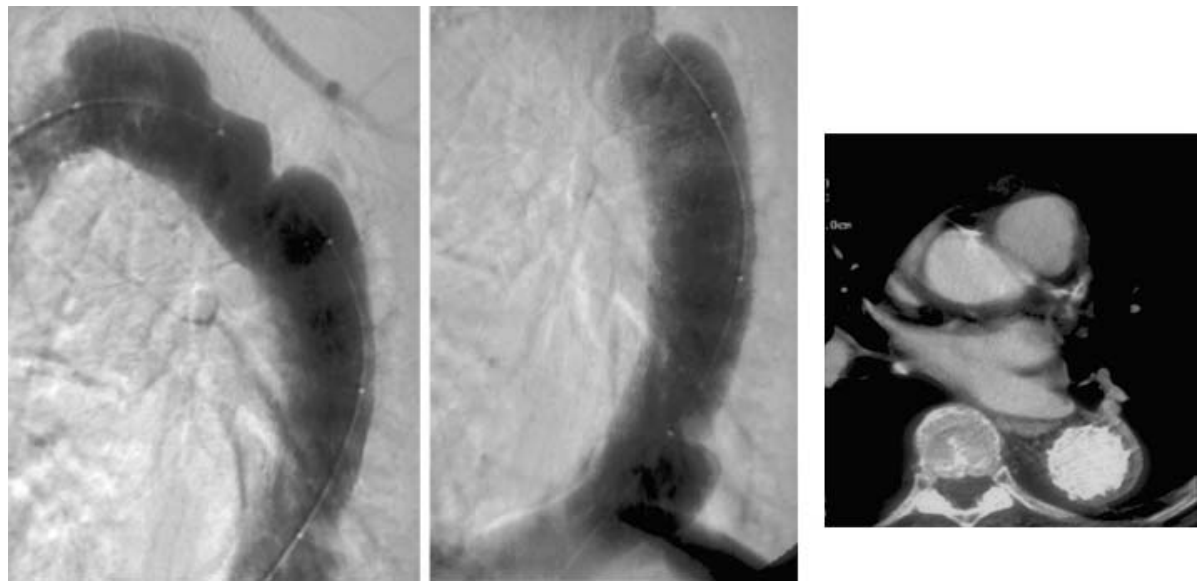

Figure 4 Evolution of type B IMH preand post-endovascular management of associated ulcer. Follow up aortogram 10 weeks after intervention shows intimal erosion and IMH cavitation proximal and distal to the device; however, no abnormalities are evident angiographically or on CT scans in the segment where the stent-graft was placed.
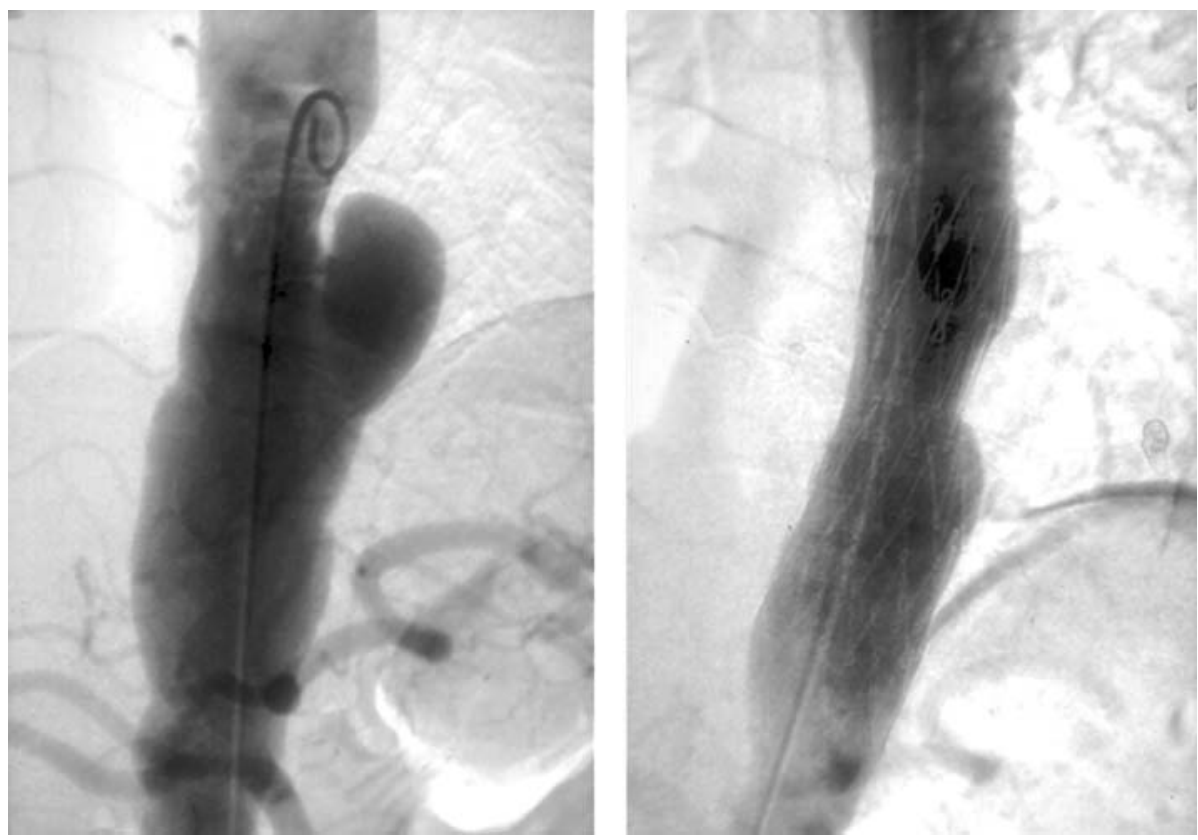

Figure 5 Stent-graft management of type B IMH and associated giant aortic ulcer. Arteriograms performed before and after stent-graft placement.

arch or descending aorta, watchful waiting and aggressive antihypertensive treatment appear a safe strategy; however, frequent follow up imaging evaluations for evidence of intimal erosion or disease progression is required and a low threshold for intervention should be maintained if symptoms recur $^{13}$ (figs 2,3 , and 4 ). This conservative approach to type $\mathrm{B}$ IMH is supported by studies that document similar survival statistics for medical treatment and surgical repair in the
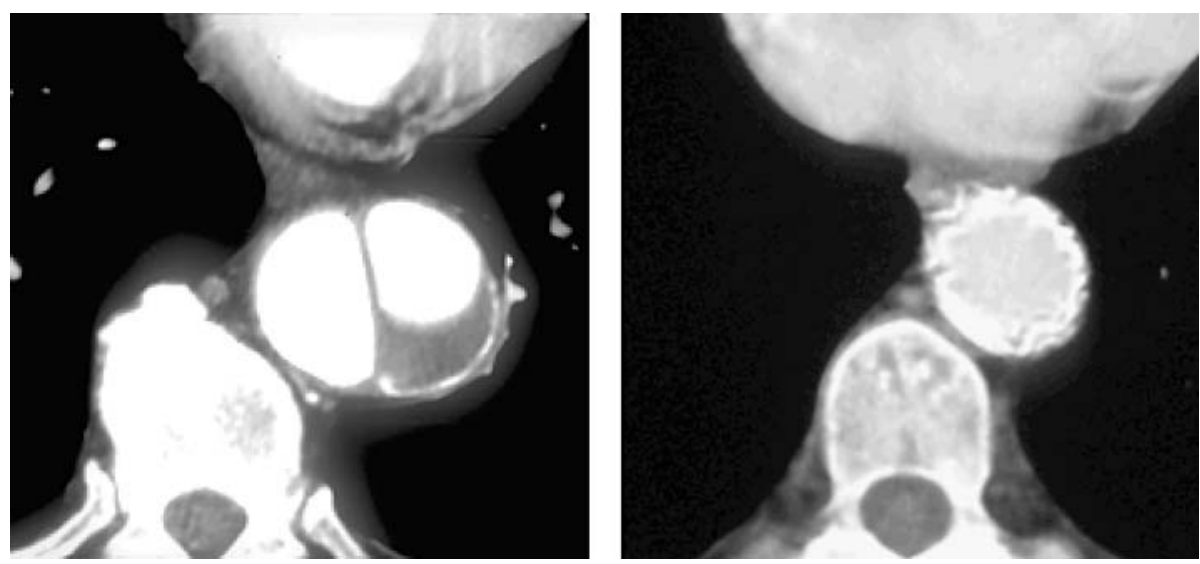

Figure 6 Stent-graft management of type B IMH and associated giant aortic ulcer. CT scans at the identical axial level show resorption of IMH and ulcer following endovascular treatment. 
setting of descending thoracic IMH. ${ }^{569}$ In a series of 53 cases of type B IMH, Harris and colleagues reported similar survival results for 33 patients managed medically and 20 who underwent surgery $\left(91 \%\right.$ and $80 \%$, respectively). ${ }^{9}$ Thus, although the cumulative risk of a conservative management policy is not defined precisely for type B IMH, there exists no clear advantage to an operative strategy.

There are clinical scenarios, however, that are considered exceptions to this approach. Most notably, the presence of a penetrating aortic ulcer in an acutely symptomatic patient or an unstable or enlarging distal ulcer associated with type B IMH should undergo more aggressive treatment. ${ }^{1}$ Expeditious surgical intervention is recommended in this setting; however, conventional open repair requiring graft interposition is associated with high morbidity and mortality rates, especially in this complicated setting in patients that typically are not ideal operative candidates because of advanced age and/or coexisting medical diseases. Consequently, less invasive strategies that rely on the endovascular placement of stent-grafts to cover the ulcer and some extent of the IMH have been recently investigated with promising initial results, including a lack of early pseudoaneurysm formation in the treated aortic segment (figs 5 and 6). Indeed the endovascular approach may have considerable advantages in this disease compared with conventional open surgical repair. ${ }^{15}$ Application of this minimally invasive technology to cases of type B IMH without associated intimal disruption or ulcer has not been reported. As additional correlative studies from larger clinical series than previously published and the results of new therapies are reported in the literature, there is an opportunity to refine management approaches to IMH and establish treatment guidelines that will improve outcomes and benefit patients beyond what is achieved currently.
Correspondence to: Michael D Dake, MD, Stanford University School of Medicine, Room H3647, 300 Pasteur Drive, Stanford, CA 94305, USA; mddake@stanford.edu

\section{REFERENCES}

1 Ganaha F, Miller DC, Sugimoto K, et al. The prognosis of aortic intramural hematoma with and without penetrating atherosclerose ulcer: a clinical and radiological analysis. Circulation 2002;106:342-8.

2 Robbins RC, McManus RP, Mitchell RS, et al. Management of patients with intramural hematoma of the thoracic aorta. Circulation 1993:II1-10.

3 Nienaber CA, von Kodolitsch Y, Petersen B, et al. Intramural hemorrhage of the thoracis aorta: diagnostic and therapeutic implications. Circulation 1995;92:1465-72.

4 Nienaber CA, Sievers HH. Intramural hematoma in acute aortic syndrome: more than one variant of dissection? Circulation 2002;106:284-5.

5 Shimizu H, Yohino H, Udagawa H, et al. Prognosis of intramural hemorrhage compared with classic aortic dissection. Am J Cardiol 2000;85:792-5.

6 von Kodolitsch Y, Csösz S, Koschyk DH, et al. Intramural hematoma of the aorta: predictors of progression to dissection and rupture. Circulation 2003;107:1158-63.

7 Murray JG, Manisali M, Flamm SD, et al. Intramural hematoma of the thoracic aorta: MR image findings and their prognostic implications. Radiol 1997; 204:349-55.

8 Song JK, Kim HS, Kang DH, et al. Different clinical features of aortic intramural hematoma versus dissection involving the ascending aorta. J Am Coll Cardiol 2001;37:1604-10.

9 Harris KM, Braverman AC, Gutierrez FR, et al. Transesophageal echocardiographic and clinical features of aortic intramural hematoma. J Thorac Cardiovasc Surg 1997;114:619-26.

10 Moriyama Y, Yotsumoto G, Kuriwaki K, et al. Intramural hematoma of the thoracic aorta. Eur J Cardiothorac Surg. 1998: 13;230-9).

11 Muluk SC, Kaufman JA, Torchiana DF, et al. Diagnosis and treatment of thoracic aortic intramural hematoma. J Vasc Surg 1996;24:1022-9.

12 Sueyoshi E, Matsuoka Y, Sakamoto I, et al. Fate of intramural hematoma of the aorta: CT evaluation. J Comput Assist Tomogr 1997;21:931-8.

13 Oliver TB, Murchison JT, Reid JH. Serial MRI in the management of intramural hemorrhage of the thoracic aorta. Br J Radiol 1997:70:1288-90.

14 Kaii S, Nishigami K, Akasaka T, et al. Prediction of progression or regression of type A aortic intramural hematoma by computed tomography. Circulation 1999;100(suppl II):II-281-6

15 Schoder M, Grabenwöger M, Hölzenbein T, et al. Endovascular stent-graft repair of complicated penetrating atherosclerotic ulcers of the descending thoracic aorta. J Vasc Surg 2002;36:720-6.

\section{FROM BMJ JOURNALS}

\section{Endothelial dysfunction in young patients with rheumatoid arthritis and low disease activity}

G Vaudo, S Marchesi, R Gerli, R Allegrucci, A Giordano, D Siepi, M Pirro, Y Shoenfeld, G Schillaci, E Mannarino

Please visit the Heart website [www.heartinl. com] for a link to the full text of this article.
Background: Rheumatoid arthritis (RA) is associated with an increased risk of cardiovascular disease. Endothelial dysfunction represents the earliest stage of atherosclerosis.

Objective: To evaluate the influence of chronic inflammatory state on endothelial function in patients with RA by measuring endothelial reactivity in young patients with RA with low disease activity and without traditional cardiovascular risk factors.

Methods: Brachial flow mediated vasodilatation (FMV), assessed by non-invasive ultrasound, was evaluated in 32 young to middle aged patients with RA (age $\leqslant 59$ years), with DAS $28 \leqslant 3.2$ and without overt cardiovascular disease, and in 28 age and sex matched controls.

Results: Mean (SD) FMV was significantly lower in patients than in controls $(3.2(1.3) \% v$ $5.7(2.0) \% ; \mathrm{p}<0.001)$, inversely related to low density lipoprotein cholesterol $(r=-0.45$, $\mathrm{p}<0.05)$ and $\mathrm{C}$ reactive protein $(\mathrm{CRP})$, expressed as the value at the moment of ultrasound evaluation $(r=-0.44, \mathrm{p}<0.05)$, as the average of CRP levels evaluated at different times during the disease $(r=-0.47, \mathrm{p}<0.05)$, or as the average of $\geqslant 4$ determinations multiplied by the disease duration $(r=-0.40, \mathrm{p}<0.05)$. In a multivariate regression model, a lower brachial flow mediated vasodilatation was independently predicted by low density lipoprotein cholesterol $(\beta=-0.40, \mathrm{p}<0.05)$, average CRP levels multiplied by the disease duration $(\beta=-0.44, p<0.05)$, and brachial artery diameter $(\beta=-0.28, p<0.05)$.

Conclusions: Young to middle aged patients with RA with low disease activity, free from cardiovascular risk factors and overt cardiovascular disease, have an altered endothelial reactivity that seems to be primarily related to the disease associated chronic inflammatory condition.

A Annals of the Rheumatic Diseases 2004;63:31-35. 